\title{
Triggering for Submaximal Exercise Level in Gastric Exercise Tonometry: Serial Lactate, Heart Rate, or Respiratory Quotient?
}

\author{
Johannes A. Otte, MD, PhD • Ellie Oostveen, PhD • Peter B. F. Mensink, MD, PhD • \\ Robert H. Geelkerken, MD, PhD · Jeroen J. Kolkman, MD, PhD
}

Received: 24 September 2006 / Accepted: 16 November 2006 / Published online: 24 March 2007

(C) Springer Science+Business Media, LLC 2007

\begin{abstract}
Gastric exercise tonometry is a functional diagnostic test in chronic gastrointestinal ischemia. As maximal exercise can cause false-positive tests, exercise buildup should be controlled to remain submaximal. We evaluated three parameters for monitoring and adjusting exercise levels (heart rate [HR], respiratory quotient [RQ], and serial lactate measurements) in 178 tests in both healthy volunteers and patients suspected of gastrointestinal ischemia. Exercise levels above submaximal occurred in $20 \%$ of HR-, $2 \%$ of RQ-, and 5\% of lactate-monitored tests $(P<0.05$ for HR vs. RQ and lactate). Low levels were seen in 5\% of HR-, $10 \%$ of RQ-, and $41 \%$ of lactate-monitored tests $(P<0.01$ for lactate vs. HR and RQ). High levels resulted in $43 \%$ false-positive tonometry results compared to $19 \%$ of all tests $(P<0.001)$; low levels did not result in more false negatives (5\% vs. 6\%). Although RQ monitoring yielded the greatest proportion of optimal exercise tests, serial lactate monitoring is our method
\end{abstract}

J. A. Otte, MD, PhD

Department of Internal Medicine, Medisch Spectrum Twente,

Enschede, The Netherlands

E. Oostveen, $\mathrm{PhD}$

Department of Pulmonary Physiology, Medisch Spectrum Twente,

Enschede, The Netherlands

\section{P. B. F. Mensink, MD, PhD - J. J. Kolkman, MD, PhD}

Department of Gastroenterology, Medisch Spectrum Twente,

Enschede, The Netherlands

R. H. Geelkerken, MD, PhD

Department of Vascular Surgery, Medisch Spectrum Twente,

Enschede, The Netherlands

J. A. Otte, $\mathrm{MD}, \mathrm{PhD}(\square)$

Ziekenhuis Zeeuwsvlaanderen, Wielingenlaan 2,

Terneuzen 4535, PA, The Netherlands

e-mail: jaotte@zeelandnet.nl of choice, combining optimal diagnostic accuracy, low cost, and simplicity.

Keywords Chronic gastrointestinal ischemia .

Tonometry · Gastric exercise tonometry · Diagnosis .

Exercise test $\cdot$ Exercise level

\section{Introduction}

For diagnosis in patients with suspected chronic gastrointestinal ischemia, we have previously shown that gastric exercise tonometry can be used as a functional test providing information about the adequacy of the gastrointestinal mucosal perfusion $[1,2]$. In these studies it was demonstrated that during $10 \mathrm{~min}$ of submaximal exercise, gastric ischemia occurred only in patients with splanchnic artery stenosis. An extreme exercise level may cause false-positive tests, as shown in a study in healthy volunteers, where lactate levels exceeding $8 \mathrm{mM}$ resulted in gastric ischemia in 50\% [3]. Furthermore, it may be conceivable that exercise of very low intensity can lead to false-negative results in exercise tonometry used for diagnosing chronic gastrointestinal ischemia. In order to prevent false-positive and false-negative tonometry tests, the exercise intensity should be monitored throughout the test, and if necessary, adjustments of the workload should be made in order to obtain an optimal exercise test.

Exercise intensity can be monitored by various parameters including arterial plasma lactate concentration, decrease in serum arterial base excess (BE) or bicarbonate concentration (which are both directly related to lactate level), heart rate (HR), and respiratory parameters [respiratory gas exchange ratio $(\mathrm{RQ})=$ carbon dioxide output $\left(\mathrm{VCO}_{2}\right) /$ oxygen uptake $\left.\left(\mathrm{VO}_{2}\right)\right]$ [4]. 
In this study we evaluated and compared three consecutive time periods in each of which a different parameter was used for monitoring the exercise intensity and adjusting the workload in order to obtain a submaximal exercise test. Initially HR monitoring was used; in the second period exercise intensity monitoring and adjustment were guided by monitoring of RQ. In the final period exercise intensity was monitored by serial rapid lactate measurements.

\section{Subjects and methods}

In 10 volunteers ( 5 females, 5 males; mean age, 25 years; range, 23-28 years) and 157 patients (59 males, 98 females; median age, 55 years; range, 13-82 years), 178 tonometry exercise tests were performed. The volunteers were tested as part of a more extensive study investigating the effect of two different exercise levels on gastric tonometry [3]. All patients were suspected of having symptomatic chronic gastrointestinal ischemia. Their clinical presentation was that of unexplained abdominal pain, weight loss, diarrhea, or gastric ulcers. Gastric tonometry exercise testing was performed as a diagnostic function test in addition to duplex sonography and selective angiography of the splanchnic vessels. Some of the patients in this study have been previously described in a publication by our group investigating the diagnostic potential of gastric exercise tonometry [1].

The procedure of gastric tonometry exercise testing was described in detail in a previous study [3]. In short, a standard nasogastric tonometry catheter was inserted and connected to an automated air tonometry device that was set up to measure intragastric partial carbon dioxide pressure $\left(\mathrm{PCO}_{2}\right) \mathrm{ev}-$ ery $10 \mathrm{~min}$. All subjects were studied after a fasting episode of $4 \mathrm{hr}$. Ranitidine, $100 \mathrm{mg}$, was given intravenously $90 \mathrm{~min}$ prior to exercise testing. A radial artery catheter was introduced in the nondominant arm to allow sequential arterial blood sampling.

Exercise was performed on an electromagnetically braked bicycle ergometer (Lode, Groningen, Netherlands).

Monitoring exercise intensity and adjusting the workload

The maximal workload $\left(W_{\max }\right)$ was estimated using standard criteria, i.e., age, sex, and weight [5]. The exercise episode was started at $10 \%$ of $W_{\max }$, and in the first 4-6 min the workload was increased every minute by $10 \%$ of $W_{\max }$. The workload was intended to remain constant thereafter at a submaximal exercise level. Three different approaches for monitoring the exercise intensity and - if necessary-guiding additional adjustments of the workload were evaluated in consecutive periods.

\section{First period: HR-triggered tests}

Exercise intensity was monitored by measuring HR, aiming for a HR in the last $4-5$ min of $80 \%$ of maximal predicted $\mathrm{HR}$ ( $\left.\mathrm{HR}_{\text {maxpred }}\right) . \mathrm{HR}_{\text {maxpred }}$ was calculated by the following formula: $\mathrm{HR}_{\text {maxpred }}=210-0.65$, age [6].

\section{Second period: Respiratory gas exchange ratio (RQ)-triggered tests}

The workload adjustments were guided by measurement of the RQ, aiming for an RQ of 1.0 in the last 4-5 min of the exercise episode. This RQ was chosen as it indicates the anaerobic threshold

\section{Third period: Lactate-triggered tests}

In 40 of the RQ-triggered tests serial rapid lactate measurements were done during exercise at $t=0,4,6,8$, and $10 \mathrm{~min}$. These measurements were not used for guiding the exercise intensity, but to develop a lactate-based exercise triggering protocol. From the results of rapid serial lactate measurements in the RQ-triggered period, an algorithm was developed for triggering the exercise intensity (see Table 1). We aimed for a lactate concentration of $4 \mathrm{mM}$ in the last $4 \mathrm{~min}$ of the test. In parallel with RQ triggering at an RQ value of 1.0, this lactate level resembles the anaerobic threshold, at which redistribution of blood flow patterns is initiated.

In the first two periods (HR and RQ triggering) the tests were performed at the pulmonary function department of our hospital. During these exercise tests a 12-lead electrocardiogram was recorded (Case 12; Marquette Electronics Inc., Milwaukee, WI, USA). HR was recorded every minute. Breath-by-breath oxygen uptake $\left(\mathrm{VO}_{2}\right)$ and $\mathrm{RQ}$ were measured by a respiratory gas analyzer system (Oxycon- $\alpha$; Jaeger, Bunnik, the Netherlands) and recorded every $30 \mathrm{sec}$.

In the third period (rapid serial lactate triggering) the test was performed at the gastroenterology function department; HR was monitored, but respiratory parameters were not monitored.

Arterial blood samples for determination of base excess (BE), bicarbonate (blood gas analyzer; Radiometer ABL520, Copenhagen, Denmark), and lactate (enzymatic assay; Cobas Fara; Roche Diagnostics, Branchburg, NJ, USA)

Table 1 Algorithm for lactate-guided exercise intensity adjustments

Start at $10 \%$ of predicted $W_{\max }$

Increase workload by $10 \%$ of $W_{\max }$ every minute (at $t=1,2,3,4$,

5 , and $6 \mathrm{~min}$ )

Lactate measurements at $t=4,6,8$, and $10 \mathrm{~min}$

If lactate $>3 \mathrm{mM}$, decrease workload by $10 \%$ of $W_{\max }$

If lactate not $>1 \mathrm{mM}$, increase $2 \times 10 \%$ of $W_{\max }$ 
were drawn, in parallel with the 10-min tonometry interval, before and immediately at the end of the 10-min exercise episode. Rapid serial lactate measurements were performed using a small portable lactate analyzer (Accutrend; Roche Diagnostics, Almere, the Netherlands), specifically developed for use during exercise testing. Using this device, measurement results were available within $60 \mathrm{sec}$ after blood sampling.

The tests evaluated in the present study were all aimed at a submaximal level: lactate level (or BE decrease) at or just above $4 \mathrm{mM}$ (the anaerobic threshold) and not exceeding $8 \mathrm{mM}$. After comparison with arterial lactate measurements (see Results), BE decrease during exercise was used as the parameter assessing the exercise intensity (Fig. 1). The optimal exercise level was a BE decrease of between 3 and $7 \mathrm{mM}$. Less than $3 \mathrm{mM}$ and more than $7 \mathrm{mM}$ were regarded as low and high exercise levels, respectively.

For comparing the results of HR and RQ measurements with $\mathrm{BE}$ decrease as the indicator of the exercise intensity reached, the following parameters were used: maximum RQ reached $\left(R_{\max }\right)$, total time $R Q>1\left(R_{t>1}\right)$, maximum heart rate reached $\left(H R_{\max }\right)$ as a percentage of $H R_{\text {maxpred }}$, and total time of $\mathrm{HR}>80 \%$ of $\mathrm{HR}_{\text {maxpred }}\left(\mathrm{HR}_{\mathrm{t}>80}\right)$.

\section{Statistics}

All values are given as mean \pm SD unless otherwise stated. $P$ values $<0.05$ were considered statistically significant. Correlations between lactate and $\mathrm{BE}$ and lactate and bicarbonate decrease, and between both HR and RQ parameters and exercise intensity as measured by $\mathrm{BE}$ decrease, were cal-

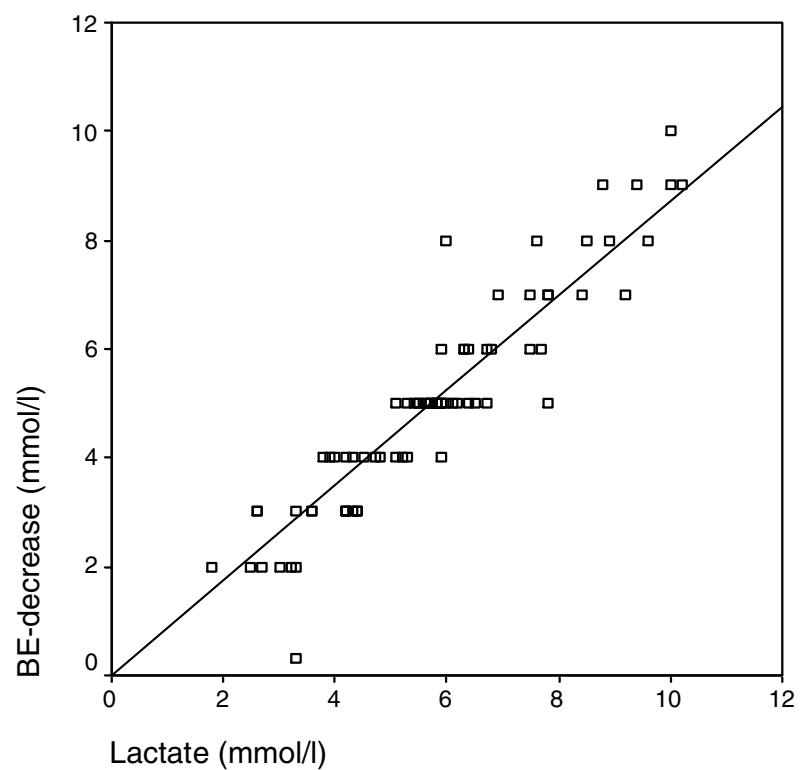

Fig. 1 Relationship between arterial lactate concentration after exercise and $\mathrm{BE}$ decrease at the end of the exercise period culated using Spearman's rank test. For comparison of HR and RQ parameters with exercise level (as divided into low, optimal, or high), one-way ANOVA with Bonferroni post hoc analysis was used. Group comparisons of the tonometry results of high and low exercise levels versus all tests and the diagnostic accuracy of tonometry in the different monitoring episodes were performed using chi-square testing.

\section{Results}

HR triggering

In 39 tests (10 in healthy volunteers and 29 in patients) HR triggering was used. In $22(56 \%)$ of these HR-triggered tests the mean HR in the last 4 min equaled the target HR of

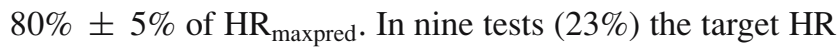
was not reached; in five of these the subjects were using $\beta$ blocking medication. In eight tests (20\%) the HR was $>85 \%$ of $\mathrm{HR}_{\text {maxpred}}$.

The resulting exercise levels as measured by BE decrease are listed in Table 2. In three tests the exercise intensity was too low. In two the target HR was not reached; one of the subjects used $\beta$-blockers. Two tonometry results were true negative; the other was false positive.

Of the eight tests with an erroneously high exercise level, in only one test was the HR too high. The other six tests in which the HR was too high resulted in the submaximal exercise levels aimed for. When analyzing the 110 tests in which both HR and RQ were recorded, only a weak correlation was observed both between $\mathrm{HR}_{\max }$ and exercise intensity and between $\mathrm{HR}_{t>80}$ and exercise intensity as measured by BE decrease ( $r=0.23$ and $r=0.20$, respectively; $P<0.05$ ). There was no significant difference for the HR-derived parameters comparing low, target, and high exercise intensity. Figure 2 displays the comparison of $\mathrm{HR}_{\max }$ for the three exercise intensity groups.

\section{RQ triggering}

In 84 tests RQ triggering was used. In $67(80 \%)$ of these tests the target RQ of 1.0 was reached while the mean RQ in the last 4 min was $1.0 \pm 5 \%$. In 11 tests $(13 \%)$ the target RQ was not reached and in 6 tests $(7 \%)$ the mean RQ in the last 4 min exceeded the target RQ by more than $5 \%$.

Of the eight tests with a low exercise level, the target RQ was not reached in four. In both tests with a too high exercise intensity, the mean RQ in the last 4 min was $>1.05$.

When analyzing all 110 tests in which RQ was monitored, positive correlations were found for comparing $\mathrm{RQ}_{\max }$ and $\mathrm{RQ}_{t>1}$ with BE decrease $(r=0.34$ and $r=0.38$, respectively; $P<0.0005)$. When divided into low, target, and high exercise intensity groups, both $\mathrm{RQ}_{\max }$ and $\mathrm{RQ}_{t>1}$ showed 
Table 2 Resulting exercise intensities for the three triggering regimes

Note. Values are given as numbers (percentage of total for triggering method). ${ }^{*} P<0.05$ and ${ }^{* *} P<0.001$ comparing the three triggering regimes.

\begin{tabular}{llll}
\hline & Exercise level & & \\
\cline { 2 - 4 } Triggering method & Low & $\begin{array}{l}\text { (BE decrease, }<3 \mathrm{mM}) \\
\text { Target }\end{array}$ & $\begin{array}{l}\text { (BE decrease, }>7 \mathrm{mM}) \\
\text { High }\end{array}$ \\
\hline HR (39 tests) & $3(8 \%)$ & $27(69 \%)$ & $9(23 \%)^{*}$ \\
RQ (84 tests) & $8(10 \%)$ & $74(88 \%)$ & $2(2 \%)$ \\
Lactate (55 tests) & $23(41 \%)^{* *}$ & $29(53 \%)^{*}$ & $3(5 \%)$ \\
\hline
\end{tabular}

significant differences among the three groups $(P<0.0005$; one-way ANOVA with Bonferroni post hoc analysis). Figure 3 displays the comparison of $\mathrm{RQ}_{\max }$ for the three exercise intensity groups.

\section{Lactate triggering}

In 55 tests lactate triggering was used. In 26 of these tests the target lactate level of $4.0 \mathrm{mM}$ in the last 2-4 min was not reached. This resulted in a BE decrease below $3 \mathrm{mM}$ in 19 tests. In two tests the rapid lactate measurements exceeded $8 \mathrm{mM}$ at $t=6$ or $8 \mathrm{~min}$. In one, the resulting exercise level was too high (BE decrease $>7 \mathrm{mM}$ ). Of the 27 tests in which the target lactate levels were reached (at least $4 \mathrm{mM}$ at $t=6$ or $8 \mathrm{~min}$, and remaining below $8 \mathrm{mM}$ ), the resulting exercise levels were too low in 7 and too high in 2.

Arterial lactate vs BE decrease and bicarbonate decrease

In 78 tests both arterial lactate levels and blood gas parameters were obtained. A high degree of correlation of both $\mathrm{BE}$ decrease and bicarbonate decrease with lactate level after exercise was found: $r=0.93$ and $r=0.89$, respectively $(P<0.0005)$. Figure 1 displays the relation between arterial lactate and $\mathrm{BE}$ decrease after exercise. Mean paired difference between lactate and BE decrease was 0.7 (SD,

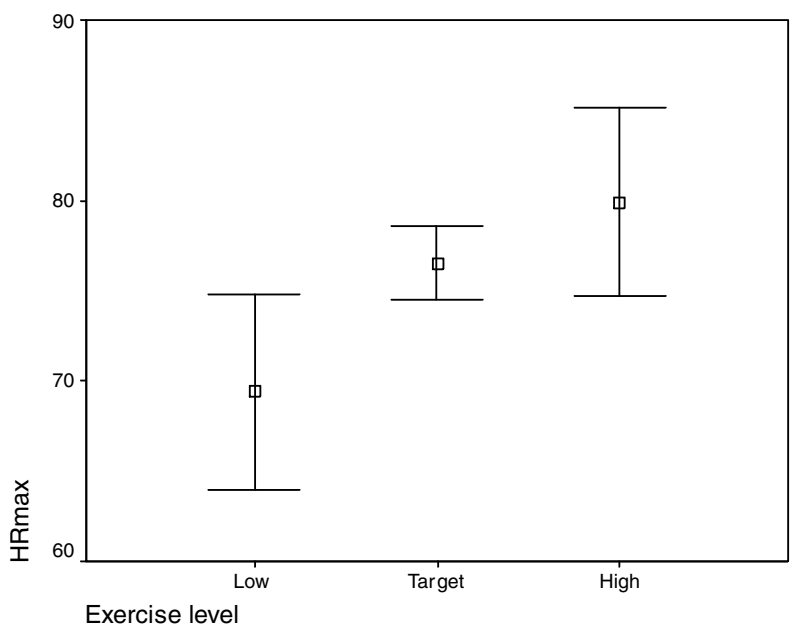

Fig. $2 \mathrm{HR}_{\max }(95 \%$ confidence interval) at low, target, and high exercise levels $\mathrm{HR}_{\max }$ is expressed as percentage $\mathrm{HR}_{\text {maxpred }}$
0.7) $\mathrm{mM}$, and that between lactate and bicarbonate decrease was $1.4(\mathrm{SD}, 0.9) \mathrm{mM}$.

Comparison accuracy of exercise tonometry at low, target, and high exercise levels

As shown in Table 1, lactate triggering resulted in significantly more low exercise levels compared to the other two regimes $(P<0.001)$.

False-negative tonometry results were found in 10 of all 178 tests $(6 \%)$. Low exercise levels did not result in an increase in false-negative tonometry tests: in only 2 of $10(5 \%)$ false-negative tests was the exercise level too low; 1 test was RQ-triggered, the other lactate-triggered.

Tonometry results were false positive in 34 of all 178 tests $(19 \%)$. Of the 14 tests with a too high exercise level, in $6(43 \%)$ the tonometry tests were false positive $(P<0.001$ comparing high exercise level vs. all tests). Three of these tests were HR-triggered (but HR did not exceed the target HR), two RQ-triggered, and one lactate-triggered.

The sensitivity and specificity of tonometry exercise testing for gastrointestinal ischemia did not differ among the three groups ( $82 \%$ and $73 \%$, respectively).

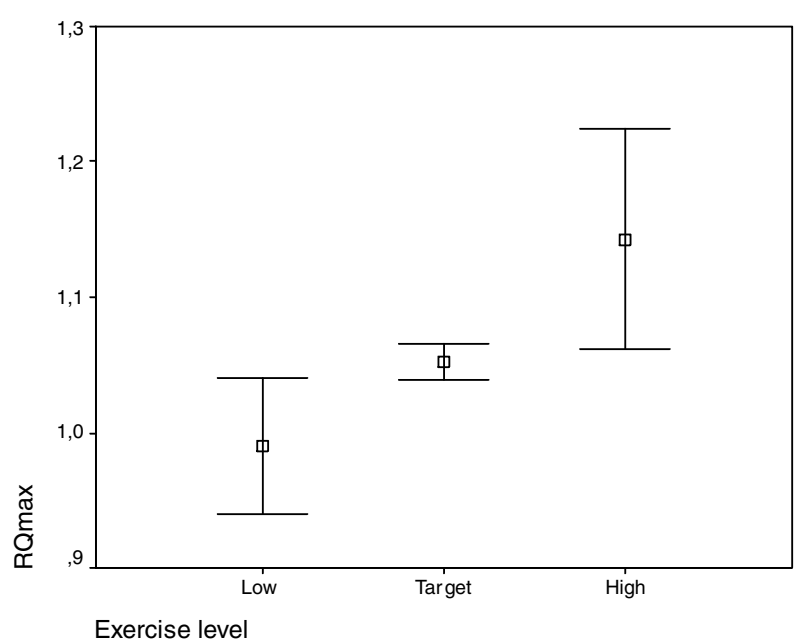

Fig. $3 \mathrm{RQ}_{\max }(95 \%$ confidence interval) at low, target, and high exercise levels 


\section{Discussion}

For optimal diagnostic accuracy, the exercise level in gastric exercise tonometry can be monitored and adjusted by RQ measuring or, alternatively, by serial arterial lactate measurement. Although the latter resulted in more tests with lower than desired exercise levels, this had no influence on diagnostic accuracy.

The greatest problem relating to exercise level in gastric exercise tonometry is the above-submaximal, or maximal, test, as this is associated with $43 \%$ false-positive tests in this study. In maximal exercise, splanchnic blood flow is severely reduced and can lead to reduction below the level required to maintain aerobic metabolism. Although data on the splanchnic vascular responses to maximal exercise have not been established, ischemia has been established frequently in these circumstances $[3,7]$. In a study on splanchnic blood flow in trained volunteers, mean superior mesenteric artery blood flow fell by $38-49 \%$ after a 10 -min exercise test at $70 \%$ of maximal workload [8]. It has been shown that the flow reduction in the celiac artery exceeds that of the superior mesenteric artery but still does not exceed about 50\% [9]. Therefore the normal splanchnic flow after submaximal exercise remains well above the normal lower level of normal splanchnic blood flow, which is estimated as approximately $30 \%$ of the normal baseline value [10]. This explains why submaximal exercise does not result in ischemia in subjects with normal splanchnic vessels. Gastric ischemia during submaximal exercise can be seen in patients with splanchnic stenoses or in patients with reduced cardiac output $[1,11]$. Thus, for practical clinical purposes $10 \mathrm{~min}$ of submaximal exercise seems adequate to distinguish between normal and pathological gastrointestinal vascular responses.

Some exercise should be performed to allow for blood flow redistribution and reduction of the splanchnic blood flow and, thus, provocation of ischemia. However, the minimally required level is currently unknown. Even with very low-intensity exercise in healthy humans a significant shift in blood flow from the abdominal viscera to the exercising muscles was observed [12]. Thus submaximal exercise as currently advocated might not be necessary, and lower lactate levels may suffice for diagnostic testing.

Triggering on RQ measurements resulted in the highest proportion of tests within the target range but has disadvantages. It requires a pulmonary function laboratory with specific devices for measurement of exhaled carbon dioxide and inhaled oxygen, making the test expensive and more complicated. The advantage of RQ monitoring in minimizing the proportion of below-target exercise level did not result in a lower number of false-negative tests in this study.

The major advantages of the serial lactate measurement are its simplicity and low cost. This rapid test can be performed by paramedic personnel and enables determination of lactate within $60 \mathrm{sec}$. This enables adequate monitoring and triggering of exercise levels. A gastric tonometry exercise test, a routine procedure at our hospital in patients suspected of chronic gastrointestinal ischemia, takes $15 \mathrm{~min}$ of a doctor's time and $2 \mathrm{hr}$ for a registered nurse. Moreover, the currently available air tonometry device allows for accurate and reproducible measurements without the hassle and problems associated with classically used saline tonometry [13].

In conclusion, by using RQ and serial lactate measurements, adequate exercise levels for gastric exercise tonometry can be achieved. Rapid lactate measurements and the presented algorithm for gastric exercise tonometry are feasible for daily clinical practice.

\section{References}

1. Otte JA, Geelkerken RH, Oostveen E, Huisman AB, Kolkman JJ (2005) Clinical impact of gastric exercise tonometry on diagnosis and management of chronic gastrointestinal ischemia. Clin Gastroenterol Hepatol 3:660-666

2. Kolkman JJ, Groeneveld ABJ, van Der Berg FG, Rauwerda JA, Meuwissen SGM (1999) Increased gastric PCO2 during exercise is indicative of gastric ischemia: a tonometric study. Gut 44:163167

3. Otte JA, Oostveen E, Geelkerken RH, Groeneveld AB, Kolkman JJ (2001) Exercise induces gastric ischemia in healthy volunteers: a tonometry study. J Appl Physiol 91:866-871

4. Wasserman K, Hansen JE, Sue DY, Whipp BJ (1987) Principles of exercise testing and interpretation. Lea \& Febiger, Philadelphia

5. Jones N (1997) Clinical exercise testing, 4th ed. W. B. Saunders, Philadelphia

6. Spiro SG (1977) Exercise testing in clinical medicine. Br J Dis Chest 71:145-172

7. Nielsen HB, Svendsen LB, Jensen TH, Secher NH (1995) Exerciseinduced gastric mucosal acidosis. Med Sci Sports Exerc 27:10031006

8. Peters HP, de Leeuw D, Lapham RC, Bol E, Mosterd WL, de Vries WR (2001) Reproducibility of ultrasound blood flow measurement of the superior mesenteric artery before and after exercise. Int $\mathrm{J}$ Sports Med 22:245-249

9. Perko MJ, Nielsen HB, Skak C, Clemmesen JO, Schroeder TV, Secher NH (1998) Mesenteric, coeliac and splanchnic blood flow in humans during exercise. J Physiol 513(Pt 3):907-913

10. Knichwitz G, Rotker J, Mollhoff T, Richter KD, Brussel T (1998) Continuous intramucosal PCO2 measurement allows the early detection of intestinal malperfusion. Crit Care Med 26:15501557

11. Krack A, Richartz BM, Gastmann A, Greim K, Lotze U, Anker SD, Figulla HR (2004) Studies on intragastric PCO2 at rest and during exercise as a marker of intestinal perfusion in patients with chronic heart failure. Eur J Heart Fail 6:403-407

12. Osada T, Katsumura T, Hamaoka T, Inoue S, Esaki K, Sakamoto A, Murase N, Kajiyama J, Shimomitsu T, Iwane H (1999) Reduced blood flow in abdominal viscera measured by Doppler ultrasound during one-legged knee extension. J Appl Physiol 86:709719

13. Kolkman JJ, Otte JA, Groeneveld AB (2000) Gastrointestinal luminal $\mathrm{PCO}_{2}$ tonometry: an update on physiology, methodology and clinical applications. Br J Anaesth 84:74-86 